\title{
LA FILOSOFIA DE LA CAUSALIDAD EN DAVIDSON
}

El trabajo de Davidson en filosofía de la causalidad, cuyos aportes centrales se encuentran contenidos principalmente en su artículo "Causal Relations"," abarca desde cuestiones acerca de la sintaxis de ciertas expresiones causales hasta problemas de orden epistemológico, pasando por algunas cuestiones ontológicas y semánticas. El tratamiento y la respuesta a los problemas de cada una de estas áreas se encuentran intimamente relacionados, en forma tal que podríamos considerarlos como un corpus de teoría.

En el presente pretendo elaborar una exposición clara de este trabajo y señalar de manera general algunos de los problemas principales que surgen en relación a ciertas tesis. En el lado expositivo respetaré, por razones de prioridad conceptual, el orden en el que Davidson aborda los problemas en el artículo citado.

La primera de estas cuestiones, dentro del ámbito de la filosofía dé la causalidad, es relativa a la forma lógica de los enunciados causales singulares (ECs, de aquí en adelante). ${ }^{2}$ Davidson aborda esta cuestión, primeramente, haciendo una revisión crítica general de algunas de las propuestas surgidas en respuesta a ella.

Una de estas propuestas afirma que los Ecs son enunciados de tipo condicional; mientras que otra tesis más general, que contiene a esta primera, sostiene que en los ECS la expresión '...causó...' es o juega el papel lógico de una conectiva sentencial, no necesariamente identificable con el condicional. La primera de estas tesis deriva, según Davidson, de la tesis de que los ECS son "instancias" particulares de leyes causales, entendidas como enunciados generales y condicionales de un cierto tipo.

Así, si la expresión '... causó. ...', en oraciones tales como 'El corto circuito causó el fuego', es una conectiva sentencial -y ésta, como su nombre lo indica, conecta o relaciona enunciados-, entonces debemos ofrecer una reformulación adecuada de

1 Davidson, "Causal Relations", en Sosa (ed.), Causation and Conditionals, 1975, pp. 82-94.

2 Para Davidson la forma lógica de una oración de un lenguaje determinado es, dicho informalmente, la estructura obtenida en el proceso de introducción de tal oración en una teoria de la verdad para el lenguaje al cual aquélla pertenece. Asl, “...debemos poner al descubierto la estructura hasta el punto en el que sea posible establecer, para una oración arbitraria, cómo su significado depende de tal estructura..." (Davidson, "The Logical Form of Action Sentences", N. Rescher (ed.), en The Logic of Decision and Action, p. 81.) 


\section{(1) El corto circuito causó el fuego}

en forma tal que las expresiones que el supuesto conectivo relaciona, sean oraciones - y no términos singulares, como 'el corto circuito' o 'el fuego'.

Davidson ofrece el siguiente enunciado como la reformulación más adecuada de (1), para los propósitos de afirmar que contiene una conectiva sentencial:

(2) El hecho de que hubo un corto circuito causó el que se diera el caso de que hubo un fuego

Supongamos que las palabras en cursiva constituyen el conectivo sentencial en cuestión. Así, sea

(3) El hecho de que $\mathrm{p}$ causó el que se diera el caso de que $\mathrm{q}$

una formulación general de la forma lógica de los Ecs - donde las palabras en cursiva constituyen un conectivo sentencial y ' $p$ ' $y$ ' $q$ ' son oraciones.

Davidson ofrece varias razones por las cuales el supuesto conectivo no es identificable con el condicional. Pero, creo yo, el argumento importante, puesto que ataca tanto a la tesis general como a esta especifica, es el que muestra que el valor de verdad de todo el enunciado causal (3) no es función o depende meramente del valor de verdad de las oraciones que contiene. ${ }^{3}$ El argumento va como sigue:

Sea la expresión ' $\hat{x}(x=x \& p)$ ' un símbolo para referirnos a la clase de todas las $x$ tales que $(x=x)$ y $p$. Si ' $p$ ' es verdadera, esta expresión referirá simplemente a la clase de todas las $x$ tales que $(x=x)$; o sea, se referirá a $\hat{x}(x=x)$. Si ' $p$ ' es falsa, aquella expresión referirá a la clase vacia.

Asf, la oración ' $\hat{x}(x=x \& p)=\hat{x}(x=x)$ ' será lógicamente equivalente a ' $p$ '; puesto que si ' $p$ ' es verdadera o falsa, la oración anterior también lo será. Pero, además, si hay una oración ' $q$ ' que tenga el mismo valor de verdad que ' $p$ ', entonces las expresiones ${ }^{\prime} \hat{x}(x=x \& p)$ ' y ${ }^{\wedge} \hat{x}(x=x \& q)$ ' serán co-referenciales.

Tenemos, asi, los siguientes pasos:

(a) El hecho de que hubo un corto circuito causó el que se diera el caso de que hubo un fuego

(B) Hubo un fuego $\leftrightarrow$ Nerón tocaba el violín - premisa - premisa

${ }^{3}$ El argumento que Davidson utiliza deriva directamente de Frege. Sin embargo, el aqui ofrecido, que es una reconstrucción de aquel, fue tomado de Mackie, The Cement of the Universe, pp. 230-231. 
(r) El hecho de que hubo un corto circuito causó el que se diera el caso de que $\hat{x}(x=x$ \& hubo un fuego $)=\hat{x}(x=x)$

- por sustitución de oraciones lógicamente equivalentes en $(\alpha)$.

(8) El hecho de que hubo un corto circuito causó el que se diera el caso de que $\hat{x}(x=x$ \& Nerón tocaba el violín $)=\hat{x}(x=x)$

- por sustitución de términos co-referenciales en $(\gamma)$.

(ع) El hecho de que hubo un corto circuito causó el que se diera el caso de que Nerón tocaba el violín

- por sustitución de oraciones lógicamente equivalentes en $(\delta)$.

Resultando que $(\varepsilon)$ es falsa.

Pero - continúa Davidson- hay dos formas de escapar al dilema que plantea este argumento: a) rechazar los principios de sustitución (de términos co-referenciales y oraciones lógicamente equivalentes) que son utilizados en él; esto es, poner en cuestión, de menos, el que la sustitutibilidad salva veritate sea una condición necesaria de la veritativo-funcionalidad de operadores o conectivos: o bien, $b$ ) "rechazar la hipótesis de que (2) da la forma lógica de (1), y con esto [rechazar] la idea de que 'causó' es un conectivo sentencial más o menos encubierto y la de que las causas son plenamente expresadas por oraciones".. Davidson acepta esta segunda opción como "totalmente preferible" respecto a la primera.

La respuesta de Davidson a la pregunta por la forma lógica de los Ecs presupone, en términos generales, la tesis de que la causalidad es una relación entre sucesos; y las consecuencias de dicha respuesta proporcionan, a su vez, una evidencia en favor de esta presuposición.

Davidson formula su respuesta haciendo, primeramente, una distinción entre dos tipos de ECS, ejemplificados por:

(4) Juan sufrió una caf́da, lo que causó que Juan sufriera una fractura de cráneo

4 Davidson, “Causal Relations", p. 86. 
(5) La caída de Juan causó la fractura en el cráneo de Juan.

Debido a que posiblemente Davidson acepta el análisis russelliano de expresiones de la forma 'el tal y tal', entonces resulta natural el hecho de que, para él, la diferencia entre (4) y (5) consiste en que (4) es verdadero si Juan sufrió más de una caída que haya causado que se fracturara el cráneo; mientras que, por otro lado, (5) sólo es verdadera, en sentido estricto, si Juan ha sufrido en su vida sólo una caída causante de la única fractura de cráneo que ha sulrido por causa de una caida.

Ahora bien, la forma lógica del enunciado (4) se expresa mediante el enunciado:

(6) Existen sucesos $e$ y $e^{\prime}$ tales que $e$ es una caída de Juan y $e^{\prime}$ es una fractura en el cráneo de Juan y $e$ causó $e^{\prime}$,

mientras que la forma lógica de (5) es la siguiente:

(7) (1e)(e es una caída de Juan) causó (ie)(e es una fractura en el cráneo de Juan)

(7) expresa algo como 'La una y sólo una caida de Juan causó la una y sólo una fractura en el cráneo de Juan'. Además, las variables $e$ y $e^{\prime}$ recorren un dominio de sucesos. Asi, es evidente que, para Davidson, la expresión '. . . causó... es un relator; es decir, es una expresión que sirve para construir oraciones a partir de términos singulares, que, en su opinión, refieren a sucesos.

A mi entender, hay dos tipos de razones en las que Davidson apoya su aceptación de una ontología de sucesos y su tesis acerca de la forma lógica de ECS, arriba enunciada. Las primeras son razones enmarcadas en el ámbito de la problemática acerca de la causalidad; las segundas, por otra parte, se hallan dentro de un campo de discusión que incluye a aquél. Ambos tipos de razones proceden en forma condicional: "Si hay cosas tales como sucesos, entonces..."; mostrándonos, por decirlo así, cuáles son las ventajas de tal suposición. Por propósitos de brevedad los formularé sólo esquemáticamente.

Dentro del segundo tipo de razones, es decir, aquellas que se formulan en un campo general de discusión, está la afirmación de Davidson de que sólo mediante la aceptación de una ontología de sucesos podemos "dar una explicación natural y aceptable de la forma lógica de ciertas oraciones cle los tipos más comunes..."; ${ }^{5}$ además de que sólo asi podemos ofrecer una explicación "extensional" de ciertas inferencias.

5 Davidson, "Individuation of Events", en N. Recher (ed.), Essajs in Honor of Carl Hempel, p. 218. 
Davidson ofrece un ejemplo de "Causal Relations" ": La inferencia de

(8) Flora se secó con una toalla rasposa

al enunciado

(9) Flora se secó con una toalla

se explica debido a que nuestra aceptación de una ontología, que incluye cosas tales como toallas, se refleja en el lenguaje. Sea ' $S(x, y, z)$ ' el predicado ' $x$ seca a $y$ con $z$ '. Si suponemos que la forma lógica de (8) es

(10) Existe una $x$ tal que $S$ (Flora, Flora, $x) \& x$ es una toalla \& $x$ es rasposa, entonces, por simple aplicación de reglas de inferencia de lógica de predicados, obtenemos:

(11) Existe una $x$ tal que $S$ (Flora, Flora, $x) \& x$ es una toalla;

es decir, obtenemos un enunciado que, siguiendo esta suposición de la forma lógica, expresa algo como 'Flora se secó con una toalla'; es decir, el enunciado (9).

Pero, continúa Davidson, si asumimos que (10) expresa la forma lógica de (8), entonces no podemos explicar la inferencia de (8) o (9) al enunciado

(12) Flora se secó

puesto que, bajo esta suposición de la forma lógica de (10) -que expresa la forma lógica de (8) —, lo más que podemos inferir es el enunciado

(13) Existe una $x$ tal que $S$ (Flora, Flora, $x$ ),

esto es, 'Flora se secó con algo', y no el enunciado deseado (12), 'Flora se secó'.

Davidson propone, para poder ofrecer una explicación unificada de ambas inferencias —o sea, de la inferencia de (8) a (9), al igual que la de (8) a (12)-, que consideremos su tesis de que la forma lógica de (8) es más eficazmente expresada por el enunciado

(14) Existe un suceso $e$, existe una $x$, tales que $e$ es un secado que Flora se hizo a sí misma y $e$ fue hecho con $x$ y $x$ es una toalla y $x$ es rasposa

6 Davidson, "Causal Relations", ed. cit., p. 88. 
Es claro que de (14) podemos inferir enunciados que expresen to que (9) y (12) afirman.

Una explicación de ciertas inferencias causales, similar a la ofrecida de (14) a (9) y (12), se obtiene igualmente en base a la suposición de que hay sucesos y de que las causas son sucesos. Así, por ejemplo, podríamos explicar la inferencia de

(15) El secado que Flora se hizo a sí misma con una toalla ayer en la noche causó el salpullido que apareció en su piel hoy en la mañana

al enunciado

(16) El secado que Flora se hizo a si misma causó el salpullido que apareció en su piel,

puesto que la forma lógica de (15) estaría dada por

(17) ( $1 e)(e$ es un secado que Flora se hizo a sí misma y $e$ fue hecho con una toalla y $t(e)=n)$ causó $(1 e)(e$ es un salpullido que apareció en la piel de Flora y $t(e)=n+i)$.

De (17), por lógica de predicados, podemos inferir

(18) (1e)(e es un secado que Flora se hizo a sí misma y $e$ fue hecho con una toalla) causó (1e)(e es un salpullido que apareció en la piel de Flora)

que expresaría la forma lógica de (16).

Ahora bien, dentro del primer grupo de razones en favor de una ontología de sucesos - es decir, razones enmarcadas en el ámbito de la filosofía de la causalidad- se localiza la argumentación de Davidson que intenta mostrar que la suposición de que las causas son sucesos nos abre la puerta para una visión más simplificada, teórica y ontológicamente, de la causalidad.

Davidson dice: Si no hay cosas tales como sucesos - ergo, las causas no son sucesos- o si, aunque aceptemos que las causas son sucesos, no distinguimos nítidamente entre sucesos y los rasgos de éstos que escogemos para describirlos, entonces, cuando digo: "La causa del incendio fue que hubo un corto circuito", se me podría replicar: "Bueno, ésta es sólo una de tantas causas: otra causa consistió en que hubo una cantidad suficiente de oxígeno; porque si no hubiese habido suficiente oxigeno, el incendio no se habría producido." Entonces resulta que el incendio fue causado por el corto circuito, la presencia de oxígeno, de material inflamable, etc. Es decir, cada causa es una 
condición que pertenece a un conjunto de condiciones que juntas fueron suficientes para la ocurrencia del incendio.

Ante esta visión de las causas como condiciones, Davidson argumenta que, cuando afirmamos: "El corto circuito causó el incendio", no decimos que el corto circuito es una de tantas condiciones que forman, a su vez, una condición suficiente del incendio. Estoy afirmando, añadiría, que ocurrió un determinado suceso, consistente en un corto circuito, y que causó el incendio. Este es un suceso individual que se caracterizo, también, por haber ocurrido en un tiempo determinado, en un lugar específico en el que había una cantidad dada de oxígeno, etc.

Así, una respuesta suficiente a la cuestión: ¿Cuál fue la causa del incendió?, bajo la suposición de que las causas son sucesos, consiste en afirmar: "El corto circuito fue la causa"; mientras que, si asumimos que las causas son condiciones, la pregunta correcta sería: ¿Cuáles fueron las causas del incendio?, y la respuesta satisfactoria consistiría en la enumeración de todas las condiciones que, juntas, fueron suficientes para su ocurrencia. Tal enumeración, a menos que se impongan ciertas restricciones, es una labor interminable.

Basándose en esta respuesta, Davidson elabora una distinción de problemas o preguntas, relativas a enunciados causales singulares:

(i) Una primera pregunta sería: ¿Cuál suceso fue la causa del incendio?

(ii) Una segunda pregunta, distinta a la anterior, se formularía así: ¿Cómo, cuándo, dónde, por qué ocurrió el suceso que, según afirmas, fue la causa del incendio?

(iii) Una tercera cuestión distinta sería: ¿En qué te basas (cuáles son tus razones) para decir que el corto circuito (ocurrido en $t$ en un lugar $l$ ) fue la causa de este incendio determinado?

Es la respuesta a esta tercera cuestión la que nos llevaría a considerar, según Davidson, cuáles descripciones del suceso que, según pretendemos, fue la causa del incendio, debemos escoger para que podamos saber, en base al conocimiento de leyes causales y de cierto enunciado que afirme la ocurrencia de este suceso señalado mediante estas descripciones, que este suceso, en efecto, fue la causa. Asi, el suceso debería tal vez describirse como aquel suceso que consistió en un corto circuito, que ocurrió en presencia de oxígeno, donde había material inflamable, etc. ( $Y$, posiblemente, algunas de estas características deberían describirse como la posesión de ciertas propiedades fisicoquímicas.)

Ahora bien, estas consideraciones llevan a Davidson a lo que podría conside- 
rarse el segundo gran tema general que le ocupa en el artículo citado, y que es el que se refiere a la relación entre los Ecs y las leyes causales.

Davidson inicia esta parte rechazando la tesis de que podemos dar un análisis de los ECs en términos de su supuesta conexión con las leyes causales. Más exactamente, Davidson niega que con ' $a$ causó $b$ ' signifiquemos que " $a$ y $b$ puedan describirse en forma tal que la existencia de cualquiera de ellas pueda mostrarse, en base a leyes causales, como una condición necesaria y suficiente de la existencia de la otra". " Sólo mencionaremos que Davidson ofrece un argumento, al parecer contundente, que muestra que este análisis sería incorrecto; puesto que, basándose en él, todo suceso $c$ puede mostrarse como condición necesaria y suficiente de la existencia de $a$ o de $b$.

Pero, aun cuando la relación entre Ecs y leyes causales no sea una relación analítica o definicional, queda pendiente, no obstante, la cuestión acerca del tipo de relación entre estas clases de enunciados.

Ahora bien, una tesis contemporánea relativa a esta cuestión es la que afirma, aproximadamente, que los ECS son "instancias" particulares de ciertas leyes causales. En relación a esta tesis, y a la luz de su análisis de la forma lógica de los ECs, Davidsun se pregunta cuál sería la reconstrucción lógica más adecuada de aquella tesis. Es decir, su pregunta se formula, más o menos, en los siguientes términos: ¿Cuál debería ser la forma lógica de cualquier ley causal para que, a partir de ella y de la premisa que afirme que un suceso de una cierta descripción (aceptable) ocurre, podamos inferir un ECS que afirme que este suceso causó o fue causado por otro? Davidson responde: "Una posibilidad que encuentro atractiva es que una ley causal completa [full-fledged] tiene la forma de una conjunción" 8 de dos enunciados, (S) y (N), cuya forma lógica se expresa a continuación:

$$
\text { (L) }\left\{\begin{aligned}
(\mathrm{S}) \quad(e)(n)((F e \& t(e)=n) \rightarrow & \\
& (\text { (G!f })(G f \& t(f)=n+\varepsilon \& C(e, f))) \\
(\mathrm{N})(e)(n)((G e \& t(e)=n+\varepsilon) \rightarrow & (\text { (马If })(F f \& t(f)=n \& C(f, e)))
\end{aligned}\right.
$$

Las variables ' $e$ ' y ' $f$ ' recorren un dominio de sucesos; ' $n$ ', un dominio de números; $F$ y $G$ son propiedades de sucesos; ' $C(e, f)$ ' es el predicado ' $e$ causa $f$ ', y ' $t$ ' es una función que asigna un número a un suceso, indicando el momento en el que éste ocurre.

Así, si tenemos que (S) y (N) expresan cuál debería ser la forma lógica de una ley causal (L), entonces vemos que de (L) y de la premisa

(P) $(\exists ! e)(F e \& t(e)=3)$

7 Davidson, op. cit., p. 90.

8 Davidson, op. cit., p. 90. 
podemos inferir el enunciado causal singular

(C) $(1 e)(F e \& t(e)=3)$ causó $(1 e)(G e \& t(e)=3+\varepsilon)$

De igual forma, si en vez de (P) tuviésemos

$\left(\mathrm{P}^{\prime}\right)(\mathrm{Gle})(\mathrm{Ge} \& \mathrm{t}(e)=3+\varepsilon)$

podriamos inferir (C), en base a (L).

\section{III}

Hasta este punto, Davidson parece comprometido sólo con la tesis de que la reconstrucción más adecuada de la idea de que los ECs son "instancias" particulares de leyes causales, es la que él propone y que hemos expuesto arriba. Sin embargo, dada su reconstrucción, ¿podríamos adscribirle la tesis de que todo enunciado causal singular verdadero está respaldado por una ley causal, en la forma en que, en su análisis, (L) respalda a (C)? Davidson, de hecho, no afirma que todo enunciado causal singular verdadero pueda inferirse lógicamente de alguna ley causal verdadera. Así, vemos que señala explícitamente:

La gran mayoría de los enunciados causales singulares, podemos estar seguros, no están respaldados por leyes, en la forma en que (C) está respaldado por $(\mathrm{L})$. $^{9}$

$\mathrm{Y}$ esto sucede así por cualquiera de las dos siguientes razones: Primera, porque, aun cuando existan una o varias leyes causales bajo el alcance de las cuales caigan los sucesos de los cuales se afirma que están causalmente relacionados en el ECS en cuestión, tales sucesos no están descritos en este ECS en forma tal que podamos saber que éstos, en efecto, caen bajo el alcance de la o las susodichas leyes; segunda, y principal, porque tales leyes no han sido formuladas. Vemos, entonces, que seguidamente Davidson afirma:

(T) La relación [entre Ecs y leyes causales], en general, es más bien ésta: si ' $a$ causó $b$ ' es verdadero, entonces existen descripciones de $a$ y $b$ 'tales que el resultado de sustituir ' $a$ ' y ' $b$ ' por ellas' en ' $a$ causó $b$ ' es implicado por premisas verdaderas de la forma (L) y $(\mathrm{P}), y$ el converso es verdadero si se estipulan ciertas restricciones adecuadas sobre las descripciones. ${ }^{10}$

Así, para Davidson, si un Ecs ' $a$ causó $b$ ' es verdadero, entonces

9 Davidson, op. cit., p. 91.

to Davidson, op. cit., pp. $91-92$. 
(i) existen descripciones de $a$ y $b$, digamos ' $(i e)(F e \& t(e)=n)^{\prime}$ y '( $(1 c)(G e$ \& $t(e)=n+i)^{\prime}$ respectivamente, tales que

(ii) existen (existirán o deben existir) una o varias leyes causales verdaderas $L_{1}, \ldots, L_{r}$, cuya forma lógica se expresa por medio del enunciado (L) —arriba formulado_, tales que

(iii) el enunciado

'( $(e)(F e \& t(e)=n)$ causó $(1 e)(G e \& t(e)=n+i) '$

—que es el el resultado de sustituir en ' $a$ causó $b$ ', los términos ' $a$ ' y ' $b$ ' por las descripciones enunciadas en (i) - es implicado por las leyes (cuya existencia se postula en (ii)) más la premisa (P), igualmente enunciada antes.

Ahora bien, ¿̇cuál es o podría ser el argumento —o evidencia- de Davidson en favor de la que hemos llamado tesis $(T)$ ? Esto es, ¿sobre qué bases Davidson podria afirmar, fundamentadamente, que de la verdad de un ECs podemos inferir que hay - habrá o debe haber- una o varias leyes causales estrictas?

Davidson, de hecho, no argumenta en favor de esta afirmación. Más aún, en base sólo a su tesis de que es muy plausible - literalmente, "una posibilidad atractiva" - la reconstrucción que propone de la idea de que los Ecs son instancias de leyes causales, él no cuenta con evidencia de peso para afirmar que la verdad de un Ecs implica la existencia de leyes causales. Esto por dos razones:

Primera, porque aunque su reconstrucción no sólo fuese plausible, sino de hecho aplicable hoy por hoy a todo caso relevante de ECs, todo lo que de la adecuación y "veracidad" de la reconstrucción se desprendería serían condicionales de la forma ( $(\mathrm{L})$ y (P) entonces (C)'; es decir, que sólo podríamos afirmar que de la verdad de una o varias leyes, y de la premisa (P), podemos inferir que un ECS es verdadero - si el Ecs en cuestión se deriva lógicamente, en efecto, de (L) y (P). No obstante, el condicional '(C) entonces (L) y (P)', que es, dicho de una forma burda y aproximada, la parte importante de la tesis ( $T$ ) que estamos revisando, no se sigue de ninguna manera de la adecuación o "veracidad" de la reconstrucción propuesta — suponiendo que lo sea.

Segunda, porque aun cuando de la adecuación de la reconstrucción se siguieran los condicionales deseados, ' $(C)$ entonces $(L)$ y $(P)$ ', tal reconstrucción, como Davidson mismo acepta, no es aplicable a la mayoría de los casos de ECS.

\section{IV}

Ahora bien, no pretendemos que Davidson sostenga que la plausibilidad -0 , incluso, la verdad- de la tesis (T) esté. apoyada en la tesis de que su re- 
construcción es adecuada o plausible. Es posible que se incline más a sostener que la tesis se introduce en vista de la necesidad de diferenciar la relación de causalidad de otras relaciones entre sucesos, principalmente relaciones temporales.

Pero ¿sostendría esto Davidson? ¿Es suficiente argumentar esto para defender $(T)$ ? Velamos ya que, por una parte, él rechaza la tesis de que un Ecs ' $a$ causó $b$ ' pueda analizarse en términos de la supuesta relación que tiene con leyes causales. $O$, dicho de otra forma, rechaza la idea de que con ' $a$ causó $b$ ' significamos que $a$ y $b$ puedan describirse en forma tal que la existencia de cualquiera de estos sucesos pueda ser demostrada en base a leyes causales y a una premisa que afirme la ocurrencia de este suceso como condición necesaria y suficiente de la existencia del otro. Vemos que, por otra parte, Davidson, textualmente, afirma: “...he abjurado del análisis de la relación causal..." 11 Pero si ha abjurado del análisis de la causalidad - ya sea en base a su conexión con leyes causales o en cualesquiéra otros términos-, si el relator '... causó...' es un "primitivo" en su teoría (como resulta claro del hecho de que aparece en el enunciado de la forma lógica de los Ecs al igual que de las leyes causales), entonces, ¿cómo diferenciar teóricamente la relación de causalidad de otras relaciones entre sucesos, principalmente la de precedencia temporal? Si no tenemos siquiera una idea sólida acerca de qué es lo distintivo de la relación causal, no tenemos manera de rechazar la tesis de que, dado que esta relación y la de precedencia temporal no presentan diferencias, es perfectamente válido decir que todos los sucesos están relacionados causalmente, puesto que parece igualmente verdadero afirmar que todos los sucesos que ocurren o han ocurrido están en relaciones de precedencia temporal.

Es en este punto donde posiblemente entraria la tesis $(T)$ de Davidson, en el sentido de que la verdad de un Ecs implica que hay - habrá o debe haberleyes causales que lo respalden; y que este hecho es lo que distingue la relación de causalidad de la de precedencia.

Otra cuestión es, diría Davidson, la de si las leyes causales que respaldan -o puedan respaldar - los Ecs tienen o no la forma lógica por él propuesta, es decir, ( $L_{1}$. Una vez decidida esta cuestión acerca de la forma lógica de las leyes causales, entonces es posible decidir la cuestión de si las leyes causales respaldan los ecs en el sentido de que, a partir de ellas y otra premisa, aquéllos, reformulados adecuadamente, se deducen lógicamente.

Vemos, pues, que esta posible vía de argumentación en favor de $(T)$ se inscribiría dentro de la teoría causal de Davidson, una de cuyas tesis consiste, burdamente, en la afirmación de que (posiblemente) no exista ningún análisis de la relación causal.

11 Davidson, op. cit., p. 90. 
¿Significa esto que, de llegar a formularse un análisis correcto de la relación causal (que no apelase a la supuesta conexión que ésta mantiene con leyes causales), la tesis ( $T$ ) resultaría falsa? $L a$ respuesta es, al parecer, negativa: Si existiese o llegase a formularse un análisis de la causalidad de este tipo, no se seguiría necesariamente que la tesis $(T)$ fuese falsa. Se seguiría, sí, que la tesis en cuestión no sería la única forma de establecer una diferencia entre la relación causal y otras relaciones entre sucesos. Esto es, se invalidaría, por lo menos hasta ese punto, esta manera de argumentar en favor de ella.

Pero, independientemente de la plausibilidad de que se llegue o no a formular un análisis correcto de la relación causal que no apele a la existencia de leyes causales, ¿qué tan eficaz es argumentar en favor de $(T)$ diciendo que ésta puede justificadamente considerarse verdadera, puesto que es necesaria para establecer una diferencia entre, digamos, las relaciones de causalidad y las de precedencia temporal? Esta manera de argumentar, formulada asi, es insuficiente en la defensa de ( $T$ ). Asl, alguien podría replicar que no es claro por qué la tesis $(T)$ es la única que podría ser útil para establecer la susodicha diferencia; que, si no existe ningún argumento en contra, una tesis distinta a $(T)$, digamos $\left(T^{\prime}\right)$, podría perfectamente servir de igual forma para este propósito; donde $\left(\mathrm{T}^{\prime}\right)$ afirmaría, aproximadamente, que la verdad de un ecs implica la existencia de ciertas leyes, estrictas o probabilistas.

¿En base a qué, sería la pregunta, podría argumentarse que la que hemos llamado tesis $\left(T^{\prime}\right)$ no establece una diferencia entre causalidad y precedencia temporal? Así, no todo par de sucesos que están en esta relación temporal se halla asociado en una cierta forma con una o varias leyes estrictas o probabilistas - i. e., como sus "instancias". Por ello, si la razón por la que se introduce la tesis $(T)$ es la de diferenciar la causalidad de otras relaciones, entonces $\left(T^{\prime}\right)-y$ quizás otras tesis alternativas posibles- podrían con igual justificación considerarse válidas.

Por ello, creo que el primer problema importante en relación a la tesis $(\mathrm{T})$ consiste en establecer por qué ésta puede considerarse la tesis correcta, la más plausible o defendible para los propósitos de diferenciar la causalidad.

\section{V}

A mi entender, hay un segundo problema en relación a la tesis enunciada, mismo que se refiere - más que a la forma en que tal tesis puede fundamentarse- a la manera en que Davidson la formula en tal pasaje. $Y$ es que, a primera vista y formulada como está, parecería que la tesis es falsa: todos los días formulamos ECS verdaderos y, no obstante, no podemos afirmar con verdad que $(a)$ existan descripciones de los sucesos involucrados causalmente tales que (b) existan leyes causales de la forma ( $L)$ y enunciados de la forma (P) tales que $(c)$ los enunciados causales singulares resultantes de la sus- 
titución de términos co-referenciales correspondientes sean implicados por los enunciados de la forma (L) y (P). Es decir, que hay Ecs verdaderos para los cuales no existen leyes causales de las cuales aquéllos puedan ser inferidos.

A mi entender, puede haber tres tipos de salidas a esta objeción (si lo es):

(a) La primera respuesta consistiría en señalar que la objeción contiene una premisa sin fundamento epistemológico, la cual consiste en la afirmación de que actualmente formulamos Ecs verdaderos. El replicante en esta línea no necesita negar que, de hecho, se han ormulado un sinnúmero de ellos. Simplemente desearía negar que el objetante tenga evidencia para afirmar que tales ECS son verdaderos; negando, con ello, el que el objetante pueda, simplemente, formular la objeción. Quizá el replicante tenga en mente, al negar que el objetante tiene "evidencia suficiente" para afirmar que los ECS son verdaderos, una conexión fuerte (no a la Davidson, que más adelante revisaremos) entre "saber que un ECS es verdadero" y "saber que una ley determinada existe y es aproximadamente verdadera".

Sin embargo, esta respuesta tiene el defecto de que, de ser adoptada en defensa de la tesis de Davidson, la dejaría aún más vulnerable. Pues ¿̨cuál podría ser ya la evidencia en la que Davidson pudiese apoyarse para afirmar que el que un ECs sea verdadero implica que existen leyes causales y descripciones...?

(b) Una segunda respuesta posible consistiría, en términos generales, en aceptar que los enunciados $\mathfrak{u}$ oraciones, como objetos de algún tipo lingüístico (y, tal vez, también las descripciones), existen; y señalando que la cuestión de si sabemos que existen o que son verdaderos (o falsos) es distinta de la anterior.

Así, diría Davidson posiblemente, el que un ecs ' $a$ causó $b$ ' sea verdadero, implica que existen los enunciados u oraciones verdaderos de la forma (L), (P) y (C), tales que a partir de (L) y (P) se deduce (C) (donde (C) es el resultado de sustituir, en ' $a$ causó $b$ ', los términos ' $a$ ' y ' $b$ ' por otras descripciones). Otra es la cuestión de si nosotros sabemos que existen, qué oraciones son y si son verdaderas. Creo que, de lo dicho por Davidson en estas líneas, ${ }^{12}$ podría inferirse que él se inclinaría más bien a aceptar esta segunda respuesta.

(c) La tercera respuesta, finalmente, procederla

(i) señalando que lo enunciado en (b) (concediendo, pues, que las oraciones existen aun cuando no las conozcamos) no es sino una manera indirecta de establecer que hay un "nexo estrecho" entre el hecho de que un suceso cause otro y el hecho de que sucedan en la realidad cierto tipo de regularidades "expresables" mediante enunciados u oraciones que tal vez tengan la forma lógica de (L) y puedan considerarse leyes;

(ii) arguyendo que, para quien no quiera suponer (por las razones que

12 Davidson, op. cit., pp. 91-92. 
sean) lo expresado arriba en $(b)-y$, me atrevería a decir, aun para algunos que acepten $(b) \ldots$, la manera $m \dot{s}$ natural de establecer la diferencia entre la relación de causalidad y la de precedencia temporal - siguiendo el espíritu de la tesis de Davidson- consiste en afirmar que, si un suceso determinado a causó otro suceso $b$, entonces todos los sucesos ocurridos en $t$ que poseen una cierta propiedad $F$ (conocida o no), que también $a$ posee, causan algún suceso, ocurrido en $t+i$, que tiene una propiedad $G$, que $b$ también posee.

Lo anterior seria tal vez una forma un poco más precisa de decir que si un suceso particular $a$ causó uno $b$, entonces ocurren cierto tipo de "regularidades" causales en la realidad, dentro de las cuales $a$ y $b$ están incluidos y que son expresables mediante leyes.

¿Por qué creemos que ésta podría ser la manera más natural de establecer la diferencia entre ambas relaciones? Porque, primero, si aceptamos (b), en vez de $(c)$, pareceria que lo que determina o caracteriza en la realidad que la relación (supuestamente causal) que se da entre dos sucesos particulares sea, en efecto, una relación causal, es, en primer término, lo que existe en una "realidad lingüística"; mientras que, aceptando $(c)$, lo que determina o caracteriza estas relaciones consiste en el que se den ciertas relaciones -causales- entre el mismo tipo de entidades que son $a$ y $b$; es decir, sucesos. Ahora bien, de la afirmación de que existe una relación de implicación entre el hecho de que se dé una relación causal particular y la existencia de ciertas regularidades "causales", en adición a la tesis de que existen enunciados u oraciones (verdaderos y/o falsos), podemos deducir la tesis expresada en (b): Que el que se dé una determinada relación causal implica que existen oraciones verdaderas de la forma (L).

No obstante, no desearía entrar en Ios problemas que surgirían de aceptar reformular (T) de la forma sugerida en $(c)$. Uno de los más serios es el de que, al parecer, no todos los tipos de "regularidad" sirven para diferenciar la causalidad, sino sólo aquellos que son expresables mediante leyes causales. Resulta, así, por lo menos a primera vista, que adoptando (c) no podríamos evitar, de cualquier forma, hacer referencia a oraciones de un cierto tipo -i.e., aquellas que consideramos "leyes".

Mi propósito aquí consiste en señalar los problemas principales que surgen en $(T) y$, secundariamente, los caminos posibles de respuesta.

\section{VI}

Ahora bien, en lo que se refiere a nuestro conocimiento de las relaciones causales, vemos primeramente que Davidson afirma:

Si esto es correcto [es decir, si es correcto lo afirmado en la tesis $(T)$ bajo consideración arriba], no se sigue que debamos ser capaces de rastrear 
una ley si sabemos que un enunciado causal singular es verdadero; todo lo que se sigue es que sabemos que debe haber una ley respaldante [covering law]. ${ }^{13}$

De igual forma, vemos que la tesis, así enunciada, puede reformularse como la tesis que afirma que

(S) si no sabemos que debe haber una ley respaldante, entonces no sabemos que un enunciado causal singular es verdadero.

¿Qué es lo que Davidson afirma con esta tesis? El primer problema que surge de ella se centra en el uso que, en su formulación, hace de la palabra 'debe'. No obstante, vemos que, por ejemplo, en otro trabajo suyo, "Actions, Reasons and Causes"," prescinde de ella, formulando la conexión establecida en (S) de la siguiente forma:

$\left(\mathrm{S}^{\prime}\right)$... para conocer que un enunciado casusal singular es verdadero... sólo es necesario saber que existe alguna ley que respalda los sucesos en cuestión. ${ }^{15}$

Vemos también que, en ese mismo artículo, Davidson afirma:

...un caso es a menudo suficiente para persuadirnos de que una ley existe y esto implica decir que estamos persuadidos, sin evidencia inductiva directa, de que una relación causal existe. ${ }^{16}$

¿Qué podemos sacar en claro de todas estas citas? ¿En qué sentido la tesis $\left(S^{\prime}\right)$ podria tomarse como (implicando) una tesis acerca de la manera en que reconocemos que un determinado caso particular de precedencia de sucesos es, en efecto, un caso de relación causal? ¿Podría, por ejemplo, atribuírsele a Davidson la tesis fuerte de que para reconocer en cada caso individual de precedencia de sucesos - $a$ y $b$, digamos - que es un caso de relación causal, es necesario haber observado antes un cierto número de casos particulares de precedencia de sucesos, en los cuales, sucesos $x$ que tienen una cierta propiedad $F$ (que $a$ posee) son seguidos siempre por sucesos $y$ que tienen una propiedad $G$ (que $b$ posee); y que, además, en aquel caso individual, sabemos que $a$ es $F$ y que $b$ es $G$ ? $O$ bien, por el contrario, ¿defendería Davidson, dado que sostiene $\left(S^{\prime}\right)$, la tesis de que la observación de cierto número de

13 Davidson, op. cit., p. 92. Subrayado mio.

14 Davidson, D., “Actions, Reasons and Causes", en Journal of Phliosophy, vol. 60 (1963), pp. 685-700.

15 Davidson, "Actions, Reasons...,", p. 699. Subrayado mlo.

16 Davidson, "Actions, Reasons...", pp. 699-700. Subrayado mío. 
casos pertinentes anteriores de precedencia invariable no es necesaria ni suficiente en ningún caso particular de reconocimiento de relaciones causales particulares? Esto es, si el mundo fuese totalmente "irregular" (si esto fuese ṕosible), ¿discerniríamos de cualquier manera los casos de simple precedencia temporal de los casos de causalidad? Davidson, a nuestro entender, no podría aceptar esta segunda tesis, por el simple hecho de que la regularidad con que se presentan los casos particulares de relaciones causales semejantes (en algún respecto) es precisamente lo que distingue la relación de precedencia de la de causalidad. Entonces, reconocer esta diferencia debe tener algo que ver con esta misma diferencia.

Pero, por lo expresado en la última cita, tampoco Davidson sostiene, de hecho, la primera tesis. Así, si "un caso es a menudo suficiente para persuadirnos de que una ley existe y... de que una relación causal existe",17 entonces no es necesario, para todo caso particular de precedencia de sucesos, el que hayamos observado con anterioridad una "regularidad" correspondiente, para poder "reconocer" que es un caso de relación causal.

En la frase anterior, he entrecomillado 'reconocer', puesto que Davidson jamás habla de 'reconocer que un caso particular es un caso de relación causal'. Las palabras que usa, en vez de 'reconocer' u 'observar', son palabras como 'persuadirnos', 'creer' y 'saber'. Quisiera aventurar la afirmación de que esto se debe, posiblemente, al hecho de que el tipo de conocimiento que tenemos de un ecs (opinión, creencia, etc.) tiene un fuerte elemento inferencial (psicológico y posiblemente lógico).

Sin entrar en la discusión de si nuestro conocimiento perceptual -visual, por ejemplo- incluye o no la elaboración de ciertas inferencias, es claro que, según Davidson, el conocimiento que tenemos de la verdad de un Ecs - cuando sabemos que dos sucesos particulares están relacionados causalmente - no es un conocimiento de tipo perceptual -o no únicamente. Así, no "vemos" que dos sucesos están relacionados causalmente, como "vemos", por ejemplo, que un suceso precede a otro $-y$, mucho menos, como vemos que los objetos tienen una forma o color determinados.

Ahora bien, en relación a persuadirnos o creer y saber que una relación causal existe -o que un Ecs es verdadero-, vemos que

a) Un caso particular, según Davidson, es con frecuencia suficiente para persuadirnos de que una relación causal existe ( $y$ de que una ley que la respalda existe).

b) Si sabemos que un enunciado causal singular es verdadero, entonces sabemos que existe una ley causal que lo respalda.

17 Davidson, op. cit., loc. cit. 
Finalmente, en relación a la pregunta: ¿qué es lo que sabe una persona cuando sabe que existe alguna ley causal que respalda un Ecs que sabe verdadero?, y asumiendo, por propósitos de brevedad, que el conocimiento es creencia verdadera justificada, tenemos que Davidson afirma:

Generalizaciones como 'si frotas un cerillo bien hecho contra una superficie preparada lo suficientemente fuerte, entonces, siendo todas las demás condiciones favorables, el cerillo se encenderá' deben su importancia, no al hecho de que podamos esperar eventualmente transformarlas en no-tendenciosas y sin excepción, sino más bien al hecho de que resumen gran parte de nuestra evidencia para creer que existen leyes causales completas respaldando los sucesos que deseamos explicar. ${ }^{18}$

Claudia Lorena García

INSTITUTO DE INVESTIGACIONES FILOSÓFicas

Universidad Nacional Autónoma de MÉxico

\section{BIBLIOGRAFIA}

Davidson, Donald, "Causal Relations", en E. Sosa (ed.), Causation and Conditionals, Oxford University Press, 1975.

—, "Actions, Reasons and Causes", en Journal of Philosophy, vol. 60 (1963).

-, "Individuation of Events", en N. Rescher (ed.), Essays in Honor of Carl Hempel, D. Reidel, Dordrecht, 1969.

-, "The Logical Form of Action Sentences", en N. Rescher (ed.), The Logic of Decision and Action, University of Pittsburgh Press, 1967.

Hume, David, A Treatise of Human Nature, Oxford University Press, 1960.

Mackie, J. L., "Causes and Conditions", en E. Sosa (ed.), Causation and Conditionals, Oxford University Press, 1975.

- The Cement of the Universe, Oxford University Press, London, 1974.

Platts, Mark, Ways of Meaning, Routledge \& Kegan Paul, London, 1979.

Stroud, Barry, Hume, Routledge \& Kegan Paul, London, 1977.

18 Davidson, "Causal Relations", p. 92. Subrayado mío. 